actions that might help readers create change (p 108).

QSHC is available on line as well as in

\section{Quality and Safety in Health Care: a time of transition}

\section{F Moss, P Barach}

\section{QHC becomes $Q S H C$}

T his issue of QHC marks its 10th anniversary-and the start of significant changes to the journal. QHC has become QSHC-Quality and Safety in Health Care. Safety is not a new topic for this journal. In the past we have included many papers relevant to risk management and the safety of health care. By adding "safety" to the title we acknowledge the growing realisation that healthcare systems too often harm the people who depend on them.

Finding ways to limit and stop the unnecessary and preventable discomfort, disability, and death directly attributable to the system of health care is a matter of urgent concern. Regular examples of such harm appear frequently in the world's newspapers. ${ }^{12}$ In this issue Millenson $^{3}$ charts the news media's influence on exposing the extent of the damage caused by health care and the importance of telling the truth to the public. Past tragedies cannot be reversed. We owe it to those who have been harmed and to future patients to work hard to understand what went wrong and how to make care safer. Liang ${ }^{4}$ reminds us, too, that honesty and disclosure to patients of all data about their care is the right policy.

Our change in title is an explicit signal of our intent to include papers that report research, ideas, and practical improvements relevant to the delivery of safer care. The distinction between quality and safety is partly a matter of semantics.

Care cannot be considered to be of high quality unless it is safe, and many of the topics considered in papers in QSHC such as team work, human factors, leadership, experiential learning and simulation, and organisational behaviour and change are relevant to all aspects of quality improvement-including safety. In this issue Carroll and Edmondson help us to understand that organisations need to be flexible, highly reliable, and willing to learn to capture complexity, and Mohr and Batalden ${ }^{6}$ remind us that all care is delivered through microsystems and that focusing on the system and the culture of care is crucial if healthcare delivery is to improve. We will continue to publish papers about the range of research relevant to developing and sustaining better and safer care. The next issue will include the first papers in a series that explores quality improvement research. Papers that describe the translation of research into practice are also important, as these may help to bridge the gap between what is known and what actually happens in routine practice.

Our transformation will include a new cover, layout, and-behind the scenesthe introduction of an electronic manuscript tracking system (see p 5) which should speed the manuscript review process. The image on the cover will change each issue and be linked to a story ( $p$ 107). In this issue the cover illustrates a systems solution to the tragedies caused by "drug swaps" — drawing up and giving the wrong drug - which even a few weeks ago caused the death of a 51 year old firefighter, father of three, in Hammond, Indiana. ${ }^{2}$ Adam Scheffler introduces an international scan of electronic information and resources about the quality and safety of care (pp 101-2) which will accompany Tom Smith's journal scan (pp 98-100). Steve Small intromartyrs of quality who have understood the problems with the quality and safety of care long before the majority (p 103). Our first hero, Ernest Codman, proposed over 70 years ago that all surgeons should collect and report their operative outcomes - an idea we are still finding difficult ( $p$ 104). And, at the end of the journal, we have introduced "Action Points", short summaries where we pose questions addressed by some of the papers in the issue and draw out possible duces a series about those heroes and a paper version. This allows easy access to papers in current and past issues of QSHC and QHC with links through to Medline for papers referred to in articles. QSHC online will be free for the next 4 months to enable the widest possible audience to access this valuable resource. We hope it will become an indispensable source of reliable information.

Although many people access the web version of the journal, in the 18 months since going on line only two people have posted electronic responses to the articles we have published. We'd like to change that and encourage more feedback from our readers. We want to exploit the enormous capacity and flexibility provided by online access by offering background material on quality and safety of care. Electronic submission and peer review of manuscripts, online publication, and links to other sources of information are just a start. In the future our website may include online publication ahead of print, online only publication, posting detailed datasets, guidelines, case studies that include quality improvement reports and near miss and adverse events, and pictures and video clips. For now we welcome readers' feedback and comments and hope that you will continue to send us papers on all aspects of quality and safety of health care (www.qualityhealthcare.com)

Qual Saf Health Care 2002;11:1

\section{Authors' affiliations}

F Moss, Editor in Chief, QSHC

P Barach, Editor, QSHC

Correspondence to: Dr F Moss, QSHC Editorial Office, BM Publishing Group, BMA House, Tavistock Square, London WC1H 9JR, UK

\section{REFERENCES}

1 Allison R. Inquest jury finds neglect by hospital led to death. The Guardian 19 January 2002, p 6

2 Firefighter hero dies after routine cardiac surgery. Chicago Tribune 11 January 2002, p

3 Millenson ML. Pushing the profession: how the news media turned patient safety into a priority. Qual Saf Health Care 2001;11:57-63.

4 Liang BA. Honesty and transparency with patients: a system for medical error disclosure. Qual Saf Health Care 2001;11:64-8.

5 Carroll JS, Edmondson A. Leading organisational learning in health care. Qual Saf Health Care 2001;11:51-6.

6 Mohr JJ, Batalden PB. Improving safety on the front lines: the role of clinical microsystems. Qual Saf Health Care 2001;11:45-50. 


\section{Shame: the elephant in the room}

\section{F Davidoff}

\section{Shame is the "elephant in the room"-something so big and disturbing that we don't even see it, despite the fact that we keep bumping into it. It is hoped that open discussion of safety issues in QSHC will remove some of the shame relating to them}

r the 1960s the results of a large randomised controlled study by the University Group Diabetes Program (UGDP) indicated that the use of tolbutamide, virtually the only blood sugar lowering agent available at the time in pill form, was associated with a significant increase in mortality rate in patients who developed myocardial infarctions. The obvious response on the part of the medical profession should have been gratitude: here was an important way to improve the safety of clinical practice. But the response was, in fact, quite different: doubt, outrage, even legal proceedings against the investigators; the controversy went on for years. Why?

An important clue to the origins of this curious anomaly surfaced at the annual meeting of the American Diabetes Association soon after the UDGP study findings were published. During the discussion a practitioner stood up and said he simply could not, and would not, accept the findings, because admitting to his patients that he had been using an unsafe treatment would shame him in their eyes. Other examples of such reactions to improvement efforts are not hard to find. Indeed, it is arguable that shame is the universal "dark side" of improvement. After all, improvement means that, however good your performance has been, it is not as good as it could be. As such, the experience of shame helps to explain why improvement - which ought to be a "no-brainer" -is generally such a slow and difficult process. ${ }^{2}$

\section{"it is arguable that shame is the universal 'dark side' of improvement"}

What is it about shame that makes it so hard to deal with? Shame, along with embarrassment and guilt, is one of the self-conscious "moral emotions"emotions that motivate moral behaviour. Current thinking suggests that shame is so devastating because it goes right to the core of a person's identity, making them feel exposed, inferior, degraded as a person; although "moral" in quality, shame is also likely to be experienced in "non-moral" situations-for example, failure in performance-and is very much dependent on what other people think; it leads to avoidance, to silence. In these respects shame differs from guilt, which is largely concerned with a particular act or behaviour, is less damaging to someone's overall sense of self-worth than shame, and motivates people to restitution, confession, and apology. ${ }^{3}$ The enormous positive power of shame is apparent in the adoption of shaming by many human rights organisations as their principal lever for social change $e^{4}$; on the flip side lies the obvious social corrosiveness of "shameless" behaviour.

Despite its potential importance in medical life, shame has received little attention in the literature on quality improvement-indeed, in the medical literature generally. A search on the term "shame" in November 2001 yielded only 947 references, a tiny fraction of the roughly seven million articles indexed in Medline. In a sense, shame is the "elephant in the room": something so big and disturbing that we don't even see it, despite the fact that we keep bumping into it.

An important exception to this medical "shame blindness" is a paper published in 1987 by the psychiatrist Aaron Lazare which reminded us that patients commonly see their diseases as defects, inadequacies, or shortcomings, and that visits to doctors' surgeries and hospitals involve potentially humiliating physical and psychological exposure..$^{5}$ Patients respond to medical shame or the fear of it by avoiding the healthcare system, withholding information, complaining, and suing. Doctors too can feel shamed in medical encounters, which Lazare suggests provokes counterhumiliation and contributes to dissatisfaction with clinical practice. Indeed, much of the extreme distress of doctors who are sued for malpractice appears to be attributable to the shame of being sued rather than to the financial losses involved. As a related issue, who can doubt that the real agenda in the controversy currently raging over mandatory reporting of medical errors is the fear of being shamed?

Doctors may, in fact, be particularly vulnerable to shame, since they are selfselected for perfectionism when they choose to enter the profession. Moreover, the use of shaming as punishment for the shortcomings of medical students, particularly during their clinical years, and for "moral errors" committed by registrars, such as lack of sufficient dedication, hard work, and a proper reverence for role obligations, ${ }^{6}$ very likely contributes further to the extreme sensitivity of doctors to shaming.

What are some of the lessons here for those working to improve the quality and safety of medical care? The first is the importance of recognising that there actually is a problem: that shame is a powerful force in slowing or preventing improvement; that unless and until shame is confronted and dealt with, progress in improvement will be slow. The second is the recognition that shame is a fundamental human emotion and is not about to go away, no matter how successful we are at handling it. Once these basic ideas are firmly rooted, the work of mitigating and managing shame can really flourish.

This work has, of course, been under way for some time. The move away from "cutting off the tail of the performance curve" - that is, getting rid of "bad apples" —and towards "shifting the whole curve" as the basic strategy in quality improvement, ${ }^{7}$ and the recognition that medical error results as much from malfunctioning systems as from incompetent practitioners, ${ }^{8}$ can be seen as important developments in this regard. These new ways of reframing the issues of improvement and safety have helped to minimise challenges to the integrity of healthcare workers and support the transformation of medicine from a "culture of blame" to a "culture of safety".

But quality improvement has another powerful tool for managing shame. Bringing issues of quality and safety out of the shadows can, by itself, remove some of the sting associated with improvement. After all, how shameful can these issues be if they are being widely shared and openly discussed (witness the recent article in Trustee magazine ${ }^{10}$ )? Here is where reports by public bodies ${ }^{8} 9$ come in, and where a journal like Quality and Safety in Health Care-with its new title, increased focus on safety as well as quality, and the BMJ Publishing Group's co-ownership of the title with IHI-can make a huge difference. More specifically, such a journal supports three major elements-autonomy, mastery, and connectedness - that motivate people to learn and improve, bolstering their competence and their sense of self-worth, 
and thus serving as antidotes to something, knowing how to do it, and shame. ${ }^{11}$

$$
\begin{aligned}
& \text { "Bringing issues of quality } \\
& \text { and safety out of the } \\
& \text { shadows can remove some } \\
& \text { of the sting associated with } \\
& \text { improvement" }
\end{aligned}
$$

The autonomy under consideration here is not the ugly variety of blind selfsufficiency that isolates and divides; rather, it is the sense that the learning and the improvement belong to the learner, rather than being imposed from outside. Getting the information on improvement that they need, when they need it, and out of their own dedicated journal certainly supports the readers' sense of autonomy. High quality, theoretically grounded, practical journal content can certainly contribute to the mastery of the readers-knowing how to do it well. And knowing as they read their journal that hundreds or thousands of like-minded people are reading the same material at roughly the same time certainly creates a sense of connectedness-instant community, if you will.

May Quality and Safety in Health Care live long and prosper, and may it help to capture and tame the elephant in the room. It would be a real shame if it didn't.

Qual Saf Health Care 2002;11:2-3

Correspondence to: Professor F Davidoff, 143 Garden Street, Wethersfield, CT 06109, USA; fdavidoff@mail.acponline.org;

andoff@earthlink.net

\section{REFERENCES}

1 Davidoff F. Mirror, mirror. Medicine enters the self-assessment era. In: Who has seen a blood sugar? Reflections on medical education. Philadelphia: American College of Physicians, 1996: 58-62.
2 Rogers EM. Diffusion of innovations. New York: Free Press, 1999

3 Eisenberg N. Emotion, regulation, and moral development. Annu Rev Psychol 2000:51:665-97.

4 Davidoff F. Changing the subject: ethical principles for everyone in healthcare. Ann Intern Med 2000;133:386-9.

5 Lazare A. Shame and humiliation in the medical encounter. Arch Intern Med 1987; 147: 1653-8.

6 Bosk CL. Forgive and remember. Managing medical failure. Chicago: University of Chicago Press, 1979: 179

7 Berwick D. Continuous improvement as an ideal in health care. N Engl J Med 1989:320:53-6.

8 Kohn LT, Corrigan JM, Donaldson MS, eds. To err is human. Building a safer health care system. Washington, DC: National Academy Press, 1999.

9 Department of Health. An organisation with a memory. Report of an expert group on learning from adverse events in the NHS chaired by the Chief Medical Officer. London: The Stationery Office, 2000

10 Hoffmeier P. CEOs: no shame in self-doubt. Trustee 2001;54:21.

11 Deci EL, Ryan RM, Williams GC. Need satisfaction and the self-regulation of learning. Learning and Individual Differences 1996;8: 165-83.

\section{The end of the beginning: the strategic approach to patient safety research}

\section{G S Meyer, J M Eisenberg}

\section{Research into patient safety has undergone a period of rapid acceleration since the decision of the US AHRQ to make a specific commitment to fund research into systems for improving patient safety}

$\mathrm{P}$ atient safety is not a new issue and has been the subject of research internationally for decades. Funding for patient safety in the US has been around for some time and, in fact, work cited in the Institute of Medicine (IOM)'s landmark report ${ }^{1}$ was funded by the Agency for Healthcare Research and Quality (AHRQ). Supported research has investigated preventable adverse drug events, ${ }^{2}$ the role of systems failures in the aetiology of medical errors, ${ }^{3}$ and the effects of the healthcare workforce on safety. ${ }^{4}$ Other funding in Australia and the UK has advanced our knowledge of patient safety considerably.

The funding of these important studies, however, was not based on any strategic commitment to addressing the patient safety challenge but, instead, the approach of research funders to patient safety had been an opportunistic one.
The agencies solicited bright patient safety researchers employing sound methodology to address compelling issues. Funding was awarded on the basis of the ability to compete successfully against a wide range of healthcare issues. As a result, the number of researchers involved, the armamentarium of methodologies, and the scope of the research has been relatively limited.

\section{BACKGROUND}

In 1999 the US AHRQ made the decision to take a different, more strategic approach to patient safety research. The Agency's fiscal year 2000 budget included a specific commitment to fund research in patient safety through a modest \$2 million investment in research on systems related best practices in improving patient safety. ${ }^{5}$ It was hoped that this initial foray into funding patient safety research would slowly evolve into a sustained initiative which would gradually grow in terms of both importance and investment.

The IOM report, ${ }^{1}$ however, dramatically changed the deliberate but slow transition to a more strategic approach in funding patient research in the US. The report highlighted the urgent need to develop an evidentiary base for safety improvement through research. In response to the IOM report, the President asked the federal government's Quality Interagency Coordination Task Force (QuIC) to draft a comprehensive plan to address the issues of medical errors and patient safety. ${ }^{6}$ Both the IOM and QuIC reports called for a substantial targeted investment in patient safety research which became a reality with the appropriation of $\$ 50$ million for patient safety research in AHRQ's fiscal year 2001 budget.

\section{"Be careful what you wish for"}

The myriad of challenges in affecting the strategic transformation of patient safety research have, at times, suggested the adage of "be careful what you wish for ...". Because patient safety research was not a new field, agenda setting and the mechanisms to support research had to be cognisant of its history. Existing literature from the safety field, for example, demonstrated the value of a multidisciplinary approach to patient safety research demanding novel tactics to promote multidisciplinary teams of researchers. The relative paucity of funding had led to a situation where there were relatively few established researchers in 
Box 1 Research areas (identified in the September 2000 summit)

- Epidemiology of errors

- Infrastructure to improve patient safety

- Information systems

- Performance shaping factors

- Evidence based interventions

- Safety cultural issues

- Educational tools

the field. To meet this challenge the new strategy to funding patient safety research had to ensure an adequate funding stream for those investigators while cultivating new researchers to build future capacity.

Finally, and most importantly, it was made clear by the Congress and the public that elegant research papers in prestigious journals were not an adequate deliverable for the public investment in patient safety research. The research investment strategy had to be crafted in a manner which maximised the likelihood that the results of the research would be rapidly and effectively translated and disseminated into widespread practice. To meet that challenge, AHRQtogether with public, private, and international partners (UK, Australia) - held a unique agenda setting summit in September 2000. The summit represented a dramatic shift from traditional research agenda setting which relied on the opinions of expert researchers who were asked "what is the cutting edge of this research field?". Instead, the patient safety research summit took a user's perspective where groups that would use the products of patient safety researchincluding patients, providers, plans, purchasers, and policy makers-were asked "what are the questions which could be addressed with research which you could use to make health care safer?" This agenda (box 1) has become a touchstone for AHRQ and many of its partners. ${ }^{7}$

Transforming patient safety research into a strategic priority also required the development of novel approaches to funding research. Investment in patient safety research by AHRQ in 2001 used a portfolio of six complementary research solicitations effectively to address the varied needs of the users of patient safety research with five distinct priorities: (1) to increase our understanding of what is working in patient safety reporting, (2) to support established and developing multidisciplinary teams to generate new knowledge to enhance patient safety, (3) to examine the role of working conditions and performance shaping factors on patient safety, (4) to evaluate information technology based interventions to improve patient safety, and (5) to promote the dissemination of the results of this research through educational programmes. Contracts to plan future work on improving patient safety data systems, develop systems based measures of patient safety, and a coordinating centre whose aim is to ensure that the whole of the investment in patient safety research is much greater than the sum of its parts are other important components of the portfolio.

The implementation of the AHRQ's patient safety agenda faced a number of significant logistical challenges including the evaluation of methodological approaches which were often unfamiliar to traditional health services researchers, difficulties in developing review panels with sufficient expertise (because most established investigators responded to the research solicitations and were conflicted), and trying to judge which applications showed the greatest promise in impacting safety in the absence of standardised approaches to measurement.

Despite all of these challenges, the quantity and quality of applications to research solicitations exceeded all expectations, and much was learned about the variety of approaches which can be applied to patient safety research. The resulting portfolio of research represents an opportunity to make a quantum leap in the evidentiary base of patient safety improvement (the AHRQ website (www. ahrq.gov) has a complete listing of the grants funded under the patient safety research initiative).

\section{WHERE ARE WE TODAY AND WHERE MUST WE GO?}

The patient safety research community has much to be proud of. There is a previously unimagined support for the field, exciting new research projects and teams, international interest and, now, a new journal dedicated to reporting these exciting findings and programmes. But significant responsibilities have come with these opportunities. The momentum for patient safety research can only be maintained if the promises made are indeed kept. The research community must not only produce an enhanced evidentiary base for safety improvement, but must also ensure that these results are translated into practice.

The transformation to a strategic approach to patient safety research has undergone a period of rapid acceleration. We have reached the end of the beginning. Funding agencies around the world are looking at the US strategic approach to funding patient safety. It is rare for a research paradigm to evolve in real time, and it is incumbent upon the patient safety research community to ensure that this transformation is sustained and not ephemeral. Continued support for patient safety research demands a return on investment. Producing great research is not enough, we must improve safety. We owe it to our patients and the public.

Qual Saf Health Care 2002;11:3-4

\section{Authors' affiliations}

G S Meyer, J M Eisenberg, Centre for Quality Improvement and Patient Safety, Rockville, MD 20852, USA

Correspondence to: $\operatorname{Dr}$ G S Meyer, Director, Centre for Quality Improvement and Patient Safety, 6011 Executive Boulevard, Suite 200, Rockville, MD 20852, USA; gmeyer@ahrq.gov

The opinions and assertions contained herein are the private views of the authors and are not to be construed as official or as reflecting the views of the Agency for Healthcare Research and Quality, and the Department of Health and Human Services.

\section{REFERENCES}

1 Institute of Medicine. To err is human: building a safer health care system. Washington, DC: National Academy Press, 1999.

2 Leape LL, Brennan TA, Laird N, et al. The nature of adverse events in hospitalized patients. Results of the Harvard Medical Practice Study - II. N Engl J Med 1991;324:377-84

3 Leape LL, Bates DW, Cullen DJ, et al. Systems analysis of adverse drug events. JAMA 1995;274:35-43.

4 Kovner C, Gergen PJ. Nurse staffing levels and adverse events following surgery. Image Nurs Sch 1998;30:315-21.

5 http://www.ahrq.gov/research/nov00/ $1100 \mathrm{ra} 31 . \mathrm{htm}$

6 Eisenberg JM, Foster NE, Meyer GS, et al. Federal efforts to improve quality of care: the Quality Interagency Coordination Task Force (QuIC) Jt Comm J Qual Improv 2001;27:93-100.

7 Meyer GS, Foster N, Christrup S, et al. Setting a research agenda for medical errors and patient safety. Health Serv Res

2001;36:X-XX 
Online submission

\section{New QSHC online submission and review system: important message for all authors and reviewers}

\section{F Moss}

New online submission and review system for QSHC

F rom January 2002 QSHC will be linked into a new online submission and reviewing system. Developed by Highwire Press (CA, USA), Bench $>$ Press $(C)$ is a fully integrated electronic system which utilises the web to allow rapid and efficient submission of manuscripts. It also enables the peer review process to be conducted entirely online. QSHC is one of the first in the BMJ Publishing Group to go on line in this way; the aim-apart from saving trees-is to speed up the frequently frustrating progress of papers from submission to publication.

Authors can submit manuscripts in any standard word processing software. Standard graphic formats acceptable include .jpg, tiff, .gif, and eps. Text and graphic files are automatically converted to PDF for ease of distribution and reviewing purposes. Authors will be asked to approve their submission before it formally enters the reviewing process; once that has been done, papers will be sent to the editor and to reviewers via the web. All transactions are secure.

You can access the system by clicking on "Submit Your Manuscript Here" on the QSHC home page (http:// www.qualityhealthcare.com) or you can access Bench>Press directly at http:// submit-qhc.bmjjournals.com.

We are very excited with this new development and want to encourage authors and reviewers to use the online system where possible. It really is simple to use and should be a big improvement on the current peer review process. Full instructions can be found on Bench > Press http://submit-qhc. bmjjournals.com and QSHC online at http:// qhc.bmjjournals.com. Please contact Natalie Davies, Project Manager, ndavies@bmjgroup.com for further information.

Qual Saf Health Care 2002;11:5

Correspondence to: Dr F Moss, QSHC Editorial Office, BM Publishing Group, BMA House, Tavistock Square, London WC IH 9JR, UK

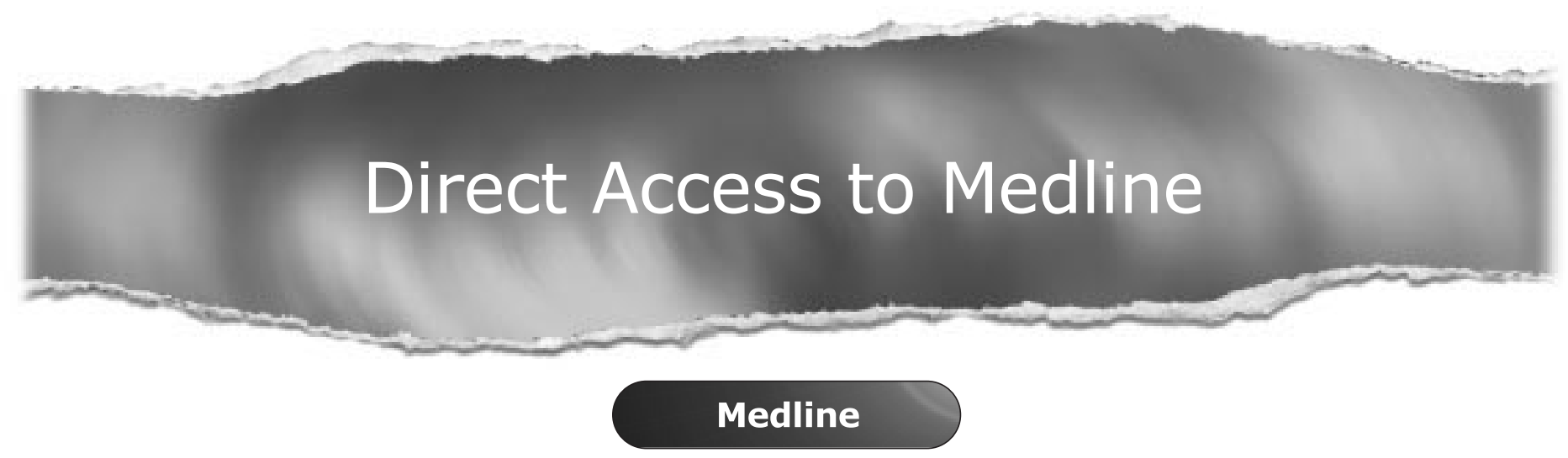

Link to Medline from the homepage and get straight into the National Library of Medicine's premier bibliographic database. Medline allows you to search across 9 million records of bibliographic citations and author abstracts from approximately 3,900 current biomedical journals.

www.qualityhealthcare.com 